\title{
Development of PET/CT and PET/MRI Patient-Information Videos in Collaboration with Patients Previously Treated for Cancer
}

\author{
Robert I. Shortman ${ }^{1,2}$, John Hoath ${ }^{1,2}$, Tina Osadolor ${ }^{2}$, Pierpaolo Inga ${ }^{3}$, Louise Roper ${ }^{1,2}$, Jamshed Bomanji ${ }^{1,2}$, \\ and Ashley M. Groves ${ }^{1,2}$ \\ ${ }^{1}$ Institute of Nuclear Medicine, University College London, London, United Kingdom; ${ }^{2}$ Biomedical Research Centre, University \\ College London Hospital, National Institute for Health Research, London, United Kingdom; and ${ }^{3}$ We Live Forever Films, London, \\ United Kingdom
}

\begin{abstract}
For patients, undergoing PET/MRI and PET/CT carries a psychologic burden that may be lessened by effective education beforehand. We devised an online resource to explain the imaging process to patients before they undergo PET/MRI or PET/CT. Methods: We produced 2 patient-information videos explaining the journey of the patient through the process of undergoing PET scanning. Actual patients were recruited to review the style and content of the videos at each stage of their production, as well as the finished product. Results: The 2 videos were produced and reviewed, and positive feedback was obtained both from the patients and from health-care providers. Conclusion: Involvement of patients in the production of the informational videos had a positive impact on the finished product and its potential benefit.
\end{abstract}

Key Words: general oncology; PET/CT; PET/MRI; public and patient involvement (PPI); YouTube; patient-information video

J Nucl Med Technol 2018; 46:26-28

DOI: 10.2967/jnmt.117.198770

$\mathbf{F}$ or patients, being scanned with PET/MRI or PET/CT carries with it the potential for anxiety, discomfort, or embarrassment, which may contribute to the overall level of psychologic burden (1). However, previous experience with scanning, or knowledge about the procedure, may allay this burden (2), and patient education before MRI has been shown to reduce anxiety (3).

In line with the findings of a previous study (4), the effect of a preparatory video before colonoscopy (5), and increased use of the Internet by people in search of health information, we formed the concept of creating

Received Jul. 5, 2017; revision accepted Sep. 26, 2017.

For correspondence or reprints contact: Robert I. Shortman, Institute of Nuclear Medicine, UCLH, 235 Euston Rd., London NW1 2BU, United Kingdom.

E-mail: robertshortman@nhs.net

Published online Oct. 17, 2017.

COPYRIGHT (c) 2018 by the Society of Nuclear Medicine and Molecular Imaging. patient-information videos for viewing online before PET/CT or PET/MRI. The aim of the videos was to orient patients to the scanning department, increase their familiarity with the procedures, and address their questions. Here, we describe our method of creating these videos, and we report feedback from patients on the final products.

\section{MATERIALS AND METHODS}

The method of creating and reviewing the videos is summarized in Figure 1.

We sought the unique perspective of patients previously scanned with $\mathrm{PET} / \mathrm{CT}$ or PET/MRI for cancer at University College London Hospitals Trust (6,7). A working panel was convened consisting of 5 such patients along with 2 research radiographers, a research nurse, a filmmaker, and an operations manager. The patients were part of a preexisting group of volunteers who assist our institution by reviewing potential projects and documentation for accessibility, or "usability," by the public. The patient volunteers were not currently under medical care at the hospital. Because this work was conducted as part of service development, rather than research, no ethical approval was sought.

First, still photographs showing various stages of the preparation and scanning processes for PET/CT and PET/ MRI were reviewed by the patients. Then, these photographs were combined into 2 videos with scripts that had been approved by the department, the footage was edited, and the videos were reviewed by both the panel and the department. Finally, after a last edit, the videos were reviewed once more by the patients.

\section{RESULTS}

Prefilming feedback indicated several aspects of the video needing improvement: there was the need to emphasize continuity of care by showing interactions between patient and radiographer, the need to show that the scanners are open at both ends, the need to indicate that there will be scanning noise, and the need to show the types of comfort aids that will be available. It was also at this stage that feedback indicated the need for 2 separate videos, one on PET/CT 


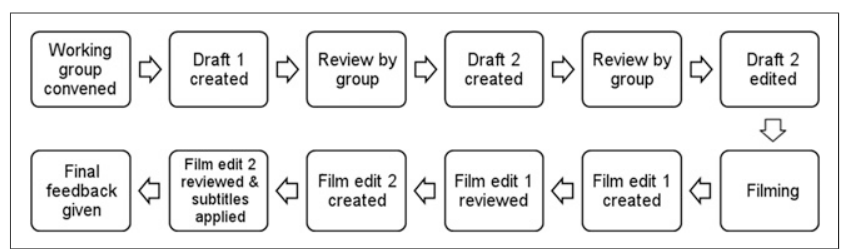

FIGURE 1. Flow diagram illustrating process of creating video.

and the other on PET/MRI, to reduce confusion; a new script was written for each.

Postfilming feedback indicated the need to show healthcare professionals performing their scanner-related duties, such as putting the patient on the scanner, performing the scan, and reviewing the resultant images (Fig. 2). Postfilming feedback also indicated a need to refine the tempo, language, and field of view.

Final-product feedback was very positive, indicating only the need to add English subtitles for individuals with impaired hearing. This positive feedback was collated and is reported in Figure 3 .

\section{DISCUSSION}

Feedback from the patients was positive. They considered that the work and effort invested had succeeded in creating a useful tool to help people who are facing not only a potentially serious disease but also a scan that they have never ex-

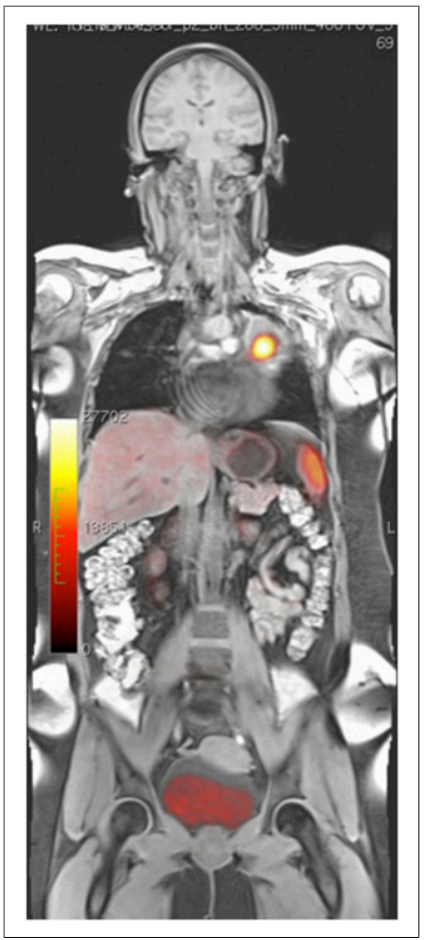

FIGURE 2. Example of PET/ MR image shown to demonstrate resultant images in informational video.

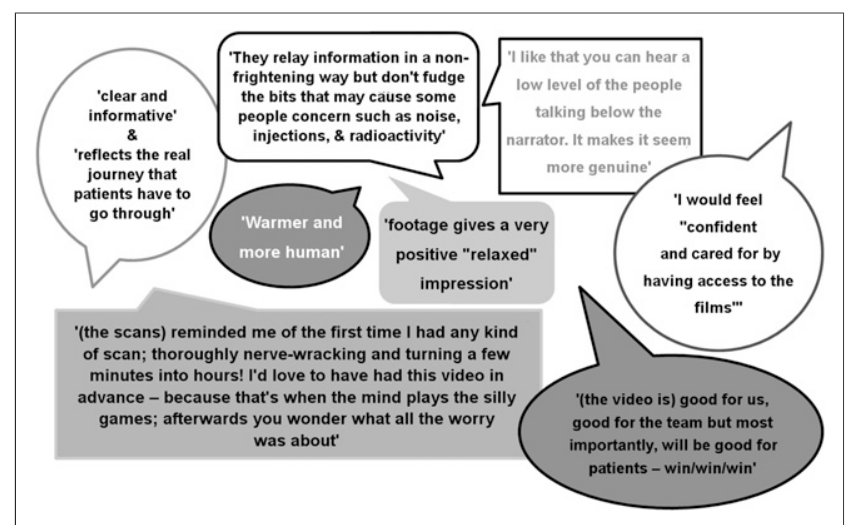

FIGURE 3. Feedback of patient panel on final versions of videos.

patients undergoing imaging, a rapidly developing field for PET/MRI.

\section{CONCLUSION}

Through extensive consultation with previous cancer patients, we created a patient-information resource to alleviate specific concerns of patients undergoing PET/CT and PET/MRI. The task now is to evaluate whether this resource alleviates patients' psychologic burden and, if so, distribute it as widely as possible to achieve the greatest benefit.

\section{DISCLOSURE}

Financial support was received from the Biomedical Research Centre, University College London Hospital, National Institute for Health Research. Pierpaolo Inga is a commercial filmmaker whose services were retained for the purposes of this project. He worked closely with the patient members of the working group to ensure their input was incorporated. No other potential conflict of interest relevant to this article was reported.

\section{ACKNOWLEDGMENTS}

We thank Jessica Skippon, Terry Emery, Maria Kacandes-Kamil, Debby Lennard, and Elizabeth LloydDehler for serving on the patient panel, as well as Libby Knowles, Victoria Moseley, James Fortune, Deena Neriman, Raymond Endozo, Gabrielle Azzopardi, Maria Machado, Marie Meagher, McKenna Roberts, and Priya Jeyanithi for helping with production of the videos. We also thank the Biomedical Research Centre, University College London Hospital, National Institute for Health Research, for their financial as well as logistical support; Phillip Brading, Professor Peter Ell, and the Governors of the University College London Hospital Charity for facilitating further financial support; and the Centre for Nurse and Midwife Led Research, University College London Hospital, for their continuing academic support and advice. 


\section{REFERENCES}

1. Bastiaannet E, Hoekstra-Weebers JE, Francken AB, Jager PL, van der Jagt EJ, Hoekstra HJ. Perception of burden experienced during diagnostic tests by melanoma patients with lymph node metastases. Melanoma Res. 2009;19: 36-41.

2. Froggatt K, Preston N, Turner M, Kerr C. Patient and public involvement in research and the Cancer Experiences Collaborative: benefits and challenges. BMJ Support Palliat Care. 2015;5:518-521.

3. Luck A, Pearson S, Maddem G, Hewett P. Effects of video information on precolonoscopy anxiety and knowledge: a randomised trial. Lancet. 1999;354:20322035 .
4. MacKenzie R, Sims C, Owens RG, Dixon AK. Patients' perceptions of magnetic resonance imaging. Clin Radiol. 1995;50:137-143.

5. Shortman RI, Neriman D, Hoath J, et al. A comparison of the psychological burden of PET-MRI and PET-CT scans and its association to initial state anxiety and previous imaging experiences. Br J Radiol. 2015;88: 20150121.

6. Vale CL, Tierney JF, Spera N, Whelan A, Nightingale A, Hanley B. Evaluation of patient involvement in a systematic review and meta-analysis of individual patient data in cervical cancer treatment. Syst Rev. 2012;1:23.

7. Vandulek C, Donkó T, Illés A, et al. Anxiety management and functional magnetic resonance imaging: should it be a priority? Ideggyogy Sz. 2015;68:318-324. 